\title{
Dynamics of Protein Elements of Hybrid Nanostructures Molecular Dynamics Simulations of Light Harvesting Peridinin-Chlorophyll $a$-Protein Model
}

\begin{abstract}
A. Jasiński, K. Mikulska, B. Krajnik, S. Mackowski and W. Nowak*
Institute of Physics, N. Copernicus University, Grudziądzka 5, 87-100 Toruń, Poland

Hybrid nanostructures are often composed of inorganic parts and "biological" ones. Optimized through million years of evolution light harvesting proteins are hard to mimic synthetically. Promising strategy in search for efficient solar cells is an attachment of selected natural protein systems to inorganic quantum dots. Such experimental hybrid structures should have improved charge separation properties. Among the most promising proteins is peridinin-chlorophyll-protein from Amphidinium carterae (PCP). It has a wide absorption spectrum $(420-550 \mathrm{~nm})$, optimized for sunlight. The dynamics of this protein, used in modern nanotechnology has been not addressed yet. In this work we present results of PCP computer modeling using a well established molecular dynamics methodology. The CHARMM27 force field parameters were prepared for this protein and all chromophore components. The system was embedded in a box of water, with proper counter ions, and a number of $10 \mathrm{~ns}$ molecular dynamics simulations were run using the NAMD code. It has been found that peridinine chromophores exhibit substantial orientational flexibility but a pair Per612 and Per613 is more rigid than the remaining two carotenoids. Orientation and dynamics of absorption and emission electric dipole moments have been also analyzed. Apparently, the architecture of PCP is not optimized for efficient Per-Chl $a$ energy transfer by the Förster mechanism. Several practical issues related to molecular dynamics simulation of similar hybrid nanostructures are discussed.
\end{abstract}

PACS: 87.10.Tf, 87.15.-v

\section{Introduction}

Hybrid nanostructures, studied vigorously due to their possible applications in new generation solar cells, are often composed of inorganic and "biological" parts. Light harvesting proteins present in photosystems of plants were highly optimized through million years of evolution and are hard to mimic synthetically. Therefore, selected crucial elements from these systems are being tested in experimental hybrid structures in order to improve charge separation properties [1]. In search for new types of photovoltaic cells peridinin-chlorophyll $a$-protein from algae (Dinoflagellate) Amphidinium carterae (PCP) is often discussed as one of the most promising light harvesting proteins $[2,3]$. PCP has a wide absorption spectrum (420-550 nm), optimized for sunlight. The solved crystal structure reveals a modular, trimeric organization of the complex [2]. Each monomeric subunit is composed of pigments grouped in two clusters (sub-monomers) related by a pseudosymmetry and encompassed by a protein environment. Each sub-monomeric cluster has one chlorophyll $a(\mathrm{Chl} a)$ surrounded by close-lying four carotenoids - peridinins (Per, see Fig. 1).

Spectral properties of PCP have been studied [4,5], the data indicate that a fast energy transfer from peridinin $\mathrm{S} 1 / \mathrm{ICT}$ excited state to Chl a $\mathrm{Q}_{\mathrm{y}}$ excited state takes place. The role of peridinins is discussed but probably they quench the Chl $a$ triplet states and thus prevent formation of dangerous singlet oxygen species [6]. The

\footnotetext{
* corresponding author; e-mail: wiesiek@fizyka.umk.pl
}

fact that PCP complex may be easily reconstructed from a recombinant protein and pigments [7] greatly enhances application potential of this system. For example, PCP having two different chlorophyll ligands ( $a$ and $b$ ) has been constructed and studied [8]. A good understanding of PCP dynamics is crucial for rational design of variants with desired technological features and good compatibility with quantum dots. In particular $\pi-\pi$ interactions Per-Chl are important in stabilizing the pigment complex [9]. Dynamics should help to understand conformational freedom of pigments in the protein matrix. Moreover, deformations on pigments modify spectral properties of PCP [10]. Inter-pigment interactions in PCP are of great interest as well, pigment position-correlation functions from molecular dynamics (MD) trajectories may help to interpret spectroscopic experiments [11]. Very recently Bonetti et al. studied ultrafast spectra of PCP and determined energy flows in electronically excited states. It has been found that peridinin molecules play different roles: ICT state mainly localizes on Per621/611 and Per623/613, the S1 state on Per622/612 and the triplet state on Per624/614 [12].

The proteins dynamics is also important from the theoretical point of view, since any calculations of the energy transfer process should take into account natural flexibility and possible reorientational changes of the chromophores. Once theoretical model of PCP is established, the role of protein/pigment mutations on spectra may be easily studied. Quite often low vibrational modes of a protein facilitate the protein function and become so-called functionally important motions. It is expected that low frequency modes may modulate spectral prop- 


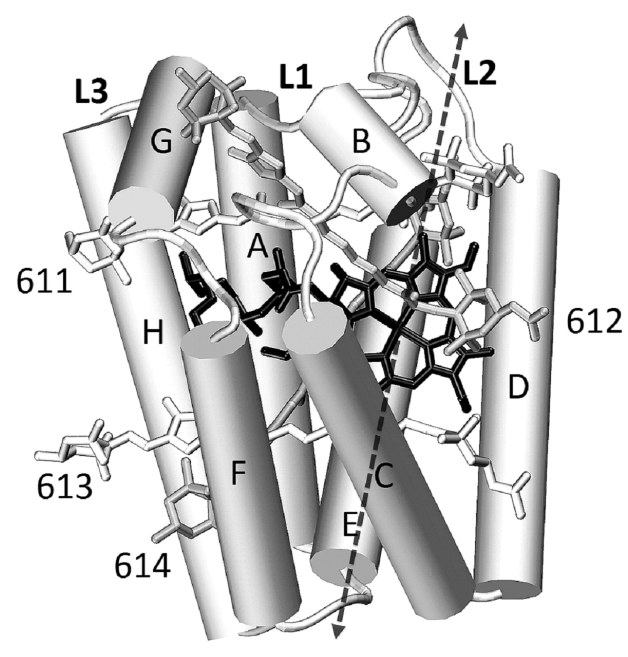

Fig. 1. The structure of a PCP complex (semi-monomer unit). Alpha helices represented by cylinders are denoted $\mathrm{A}-\mathrm{H}$, peridinin carotenoid chromophores are shown in a licorice representation, chlorophyll is indicated in black. The dashed line denotes the direction of the $S_{0}-\mathrm{Q}_{\mathrm{y}}$ transition moment in Chl $a$. For clarity J7Z lipid located in the upper right region is omitted in this figure.

erties of hybrid structures. The dynamical changes of the electrical field in locations of the chromophores affect their photophysics as well. However, the theoretical studies of PCP protein are rare. Triplet-triplet energy transfer has been calculated very recently by You and Hsu [6]. The charge transfer properties of a similar, albeit static system, has been studied quantum-mechanically in the past [13], but the classical dynamics of PCP, due to its complexity, except for a very recent conference abstract [14] and vibrational analysis of carotenoids [15], has not been addressed yet.

In order to provide a framework for studies of these dynamical aspects of the protein part of hybrid nanostructures prepared in our institute, we have developed computer models of an individual, functional submonomer of PCP. The CHARMM27 force field together with home-made parameters for pigments were used to calculate $10 \mathrm{~ns}$ MD trajectories of a complex solvated in a water box. Data were generated for the complete PCP model, an apoprotein system and for partially reconstructed PCPs. Unique information on nanosecond dynamics of PCP has been obtained which should help to develop new variants of hybrid structures.

\section{Methods}

\subsection{Parameterization}

Since PCP monomer has in its core Chl $a$ and four peridinin chromophores augmented by a large J7Z stabilizing lipid, the straightforward MD simulations using one of standard force fields are not possible. New CHARMM27 compatible parameters for prosthetic groups were therefore developed in this work, using previously established protocols $[16,18]$. The parameterization process has begun by an assignment of initial parameters for the selected model compounds extracted from the PDB 3IIS crystal structure. Parameter optimization procedure is a multistep process involving iterative recalculations based on tuned external and internal parameters. Results obtained from the CHARMM force field energy minimizations are compared to the target data from experiments or QM calculations and the parameters are manually adjusted to obtain the best agreement. The target data for charges optimization are minimum energy geometries calculated by the HF $/ 6-31 \mathrm{G}^{*}$ method, whereas as target data for bond and angle equilibrium values appropriate experimental or $a b$ initio optimized geometries were used. The gas phase $a b$ initio calculations were carried out with the Gaussian 03 program [19]. The geometries were constrained to $\mathrm{HF} / 6-31 \mathrm{G}^{*}$ optimized structure for all molecules. We used SwissParam [20] server for automatic assignment of geometry parameters and topologies for peridinins and J7Z lipid ligand. The data were derived from the Merck Molecular Force Field where dihedral angle terms had been taken "as is", and only harmonic parts of the bond, angle and improper terms were retained. Charges were assigned from $\mathrm{HF} / 6-31 \mathrm{G}^{*}$ calculations in Gaussian. Van der Waals parameters of chromophores' atoms were taken from the analogous atom types present in the CHARMM27 parameters set. The topology for Chl $a$ was built manually and parameters were adopted from well established bacteriochlorophyll $A$ parameters (Ana Damjanovic, private communication). Stability and the quality of geometry of individual ligands were tested by $1 \mathrm{~ns}$ MD simulations in a water box. 2.2. The MD protocol

The crystal structure of a single PCP domain in a complex with Chl a (PDBeChem: CLA), four peridinin (PDBeChem: PID) and one lipid (PDBeChem: J7Z) molecules was obtained from the Protein Data Bank (PDB code: 3IIS). Eight possible variants of the system, having various chromophores included or not, were prepared for simulations. For the sake of space, here we report data for the complete model and a system without J7Z lipid present. Models were solvated using a $0.8 \mathrm{~nm}$ layer of TIP3P water model in each dimension. The systems were neutralized, they include about 18200 to 20000 atoms. A cutoff of $12 \AA$ non-bonded interactions was applied during simulations. The Langevin dynamics and a Langevin piston algorithm were used to maintain the temperature at $300 \mathrm{~K}$ and a pressure of $1 \mathrm{~atm}$ [21]. MD simulations were performed using software package NAMD 2.7 with the all-atom CHARMM27 force field for the protein part and parameters for chromophores described above. Visual Molecular Dynamics (VMD) program (version 1.9) and home-made scripts were used to prepare input files and to analyze output trajectories. We performed $0.2 \mathrm{~ns}$ of water equilibration with the fix PCP model, 10000 steps of minimization, 0.35 ns of heating from $0 \mathrm{~K}$ up to $300 \mathrm{~K}$ and $0.15 \mathrm{~ns}$ equilibration of the whole system before each $10 \mathrm{~ns} \mathrm{MD}$ production run. The temperature was held constant at $300 \mathrm{~K}$ and pe- 
riodic boundary conditions were assumed. The multiple time step method was employed for the integration: time steps of 1 fs for bonded, 2 fs for short-range nonbonded, and 4 fs for long-range electrostatic forces were used. We have run twenty four 10 ns simulations for 8 different systems (the total length $240 \mathrm{~ns}$ ), thus for each system three $10 \mathrm{~ns}$ simulations were analyzed.

\section{Results and discussion}

The root mean square (rms) data show that PCP models are stable on $10 \mathrm{~ns}$, no systematic deviation is observed, possible long term process do not manifest in our simulations. MD clearly supports the experimental observation that the presence of chromophores (Chl $a$, Per) is necessary to fold this protein. Apo-protein is not stable and is much less stable in simulations than the whole PCP complex (Fig. 2).

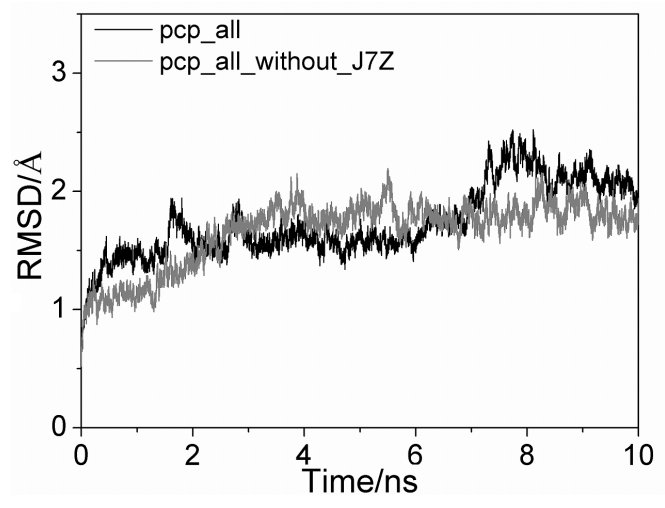

Fig. 2. The rms distances from the experimental structures calculated for all heavy atoms for two studied models of PCP: the full model and the complex with J7Z lipid removed.

Fluctuations of residues, defined as:

$$
\operatorname{RMSF}_{i}=\sqrt{\frac{1}{N_{f}} \sum_{f}\left(r_{i}^{f}-r_{i}^{\text {ave }}\right)^{2}},
$$

where $r$ denote positions of atoms from a residue $f$, are related to the Debye-Waller temperature $B$-factors

$$
B_{i}=\frac{8}{3} \pi^{2}\left(\mathrm{RMSF}_{i}\right)^{2}
$$

and correlate well with the secondary structure elements stiffness (Fig. 3). In $a$-helical regions (ACDH) the protein forms rigid scaffold. Loop regions are very flexible on $10 \mathrm{~ns}$ time scale and exhibit large fluctuations (L1, L2, L3, Fig. 3). Particularly flexible region is observed in L2. Interestingly, calculated fluctuations correlate very well with experimental Debye-Waller factors ( $B$-factors, data not shown), except regions of small helices $B$ and $G$. In crystal structures atoms of these helices have rather low values of $B$-factors, in our simulations these regions systematically are very flexible. One possible explanation of this observation is a stabilizing effect of packing forces in a PCP crystal. Perhaps L1-L3 regions (Fig. 3) play a role of hinges during reconstruction of PCP system. The folding mechanism of PCP will be studied elsewhere.

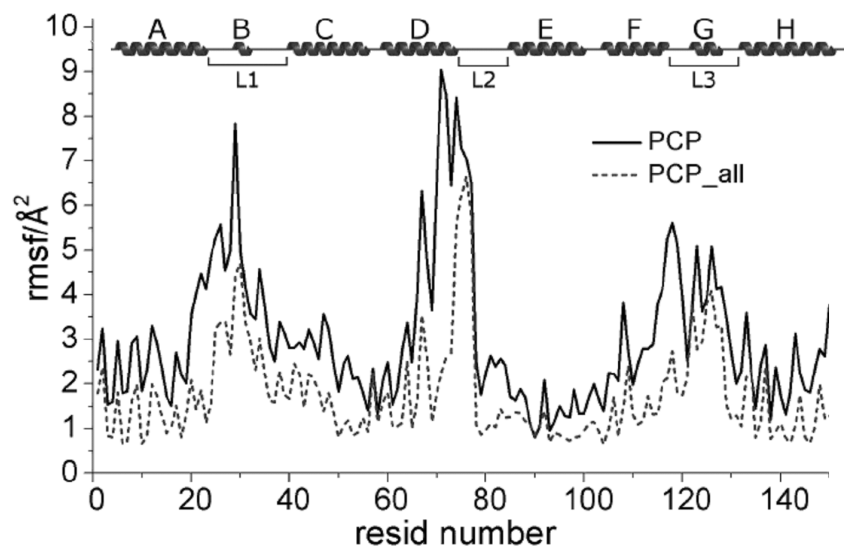

Fig. 3. Fluctuations of residues of PCP apo-protein (solid line) and the full PCP complex (dotted line). $L 1$ and $L 3$ loops regions encompass small $\alpha$ helices $B$ and $G$, respectively.

We were interested in an internal dynamics of Per chromophores and possible reorientational motions of these ligands with respect to the $\mathrm{Chl} a$ acceptor molecule. All our data show (trajectories T1, T2, T3) that long Per molecules occupy defined binding sites in the PCP complex. However, a substantial flexibility of these carotenoids is observed here. In Fig. 4 we present distributions of distances between the center of mass of Per611-614 ligands and the magnesium ion located in the middle of Chl $a$. One can easily see that these distances fluctuate, and this dynamics affects efficiency of the energy transfer in PCP. The $\mathrm{Chl} a$-Per distance may change as much as $4 \AA$ (i.e. $>30 \%$ ) - Per611. The most rigid part is Per613 binding site, these distributions of distances are rather narrow in T1-T3. A bionominal character of one distribution may indicate that an alternative binding site for Per614 is possible. The confirmation of this observation requires much longer MD simulations.

The energy transfer from Per to $\mathrm{Chl} a$ proceeds mainly through $\mathrm{S} 1 / \mathrm{ICT}-\mathrm{Q}_{\mathrm{y}}$ singlet states [12]. The distance between a donor and an acceptor molecules affects this process, though in much more complex way than the typical Förster resonance energy transfer mechanism [16]. Perhaps the exchange mechanism, due to close contacts Per-Chl $a$ play a dominant role here. However, the relative orientation of transition moments in Per donors and the Chl $a$ acceptor is of great interest. We have calculated angles between $\mathrm{Q}_{\mathrm{y}}$ transition moment axis in Chl $a$ system [22], see dashed arrow in Fig. 1, and long axes of all Per chromophores. Results are presented in Fig. 5. Detailed reorientational dynamics of each Per chromophore depends somehow on a trajectory analyzed, but certain features are common and characteristic for PCP. All absorption transitions moments (we assume that $S_{0}\left(1 A_{\mathrm{g}}\right) \rightarrow S_{2}\left(1 B_{\mathrm{u}}^{+}\right)$transition is oriented along a long axis of the conjugated systems of each Per) 


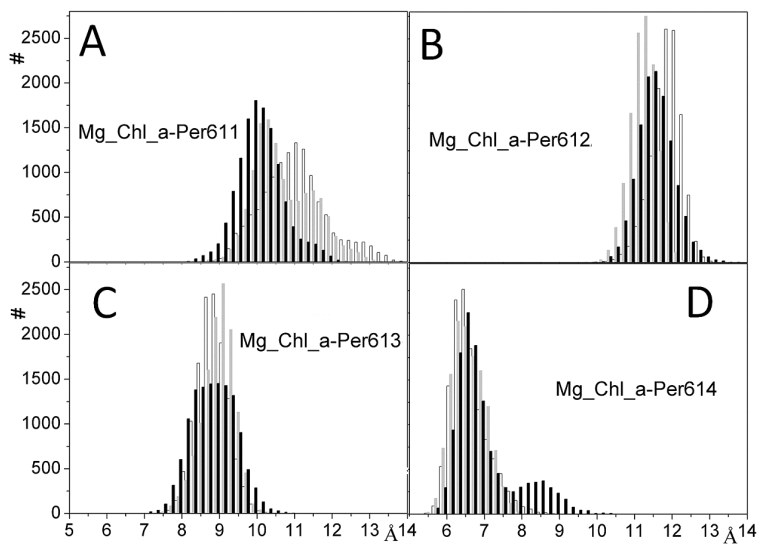

Fig. 4. Distributions of distances between the center-of-mass of peridinins and $\mathrm{Mg}^{2+}$ chlorophyll $a$ center (A)-(D). White, grey and black bars denote data extracted from $T 1, T 2$ and $T 310 \mathrm{~ns}$ trajectories of the full PCP complex, respectively.

are almost perpendicular to the absorption transition moments of Chl $a$. The angles vary from 100 to $135 \mathrm{deg}$ (see Table). Per611 and Per614 have this angle close to 100 $110 \mathrm{deg}$, while Per612 and Per613 are more tilted with respect to Chl a $\mathrm{Q}_{\mathrm{y}} \mathrm{TM}_{\mathrm{em}}$ axis: $120-130 \mathrm{deg}$. Fluctuations of these angles, showing librational motions of carotenoids, are not large, see Table. The largest flexibility is observed for Per612, the smallest for Per614. One should remember that these angles take also into account the dynamics of chlorophyll molecule as well. It is tempting to speculate that this "perpendicular" arrangement of carotenoids in PCP is not accidental and has been established by the evolution in order to minimize Per-Chl $a$ energy transfer via the Förster mechanism. In this mechanism the transfer rate depends on the orientation factor $\kappa$ which has its minimum for perpendicular $M_{\mathrm{ab}}$ and $M_{\mathrm{em}}$ vectors.

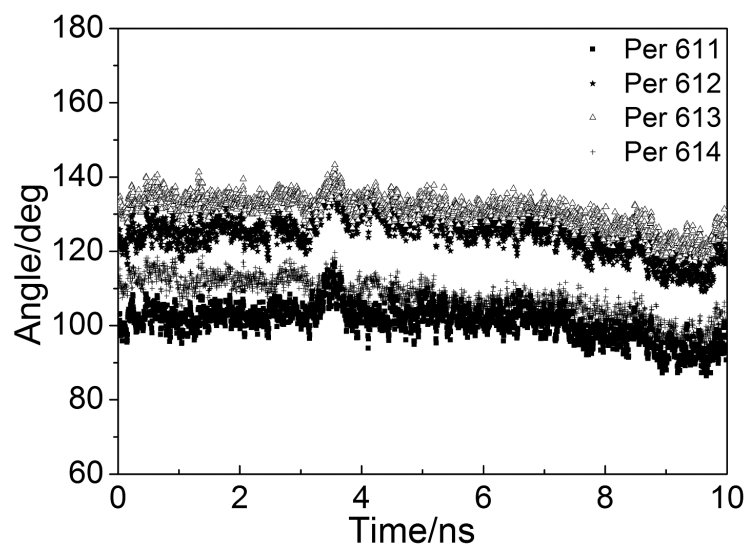

Fig. 5. An example of time evolution of angles between appropriate Per chromophores long molecular axes and the direction of the electric $\mathrm{Q}_{\mathrm{y}}-S_{0}$ transition moment in Chl $a$ moiety.

It has been recently shown that the main donor in PCP is Per611 [7] peridinin. In our MD simulations we observe that this particular ligand exhibits slightly higher internal conformational changes than the other peridinins present in the PCP semi-monomer. A certain measure of the heterogeneity of ligands' structures is their rms distance measured with respect to the reference structure. For example, for Per611we have RMSD of $1.1 \pm 0.37 \AA$ while for the other peridinins RMSD of $0.6-0.8 \AA \pm 0.17$ are observed (data obtained for trajectories without J7Z present).

TABLE

Angles (in degrees) between Per chromophores long molecular axes and the direction of the electric $\mathrm{Q}_{\mathrm{y}}-S_{0}$ transition moment in Chl $a$ moiety.

\begin{tabular}{|c|c|c|c|c|c|c|c|c|c|c|c|c|}
\hline Trajectory & \multicolumn{4}{|c|}{$\mathrm{T} 1$} & \multicolumn{4}{|c|}{$\mathrm{T} 2$} & \multicolumn{4}{|c|}{ T3 } \\
\hline Range: frames & \multirow{2}{*}{\multicolumn{2}{|c|}{$\begin{array}{l}{[0,1217]} \\
{[0,4.868]}\end{array}$}} & \multirow{2}{*}{\multicolumn{2}{|c|}{$\begin{array}{c}{[0,2500]} \\
{[0,10]}\end{array}$}} & \multirow{2}{*}{\multicolumn{2}{|c|}{$\begin{array}{c}{[0,2337]} \\
{[0,9.348]}\end{array}$}} & \multirow{2}{*}{\multicolumn{2}{|c|}{$\begin{array}{c}{[0,2500]} \\
{[0,10]}\end{array}$}} & \multirow{2}{*}{\multicolumn{2}{|c|}{$\begin{array}{c}{[0,888]} \\
{[0,3.552]}\end{array}$}} & \multirow{2}{*}{\multicolumn{2}{|c|}{$\begin{array}{c}{[0,2497]} \\
{[0,9.988]}\end{array}$}} \\
\hline Time/ns & & & & & & & & & & & & \\
\hline & Av. angle & SD & Av. angle & SD & Av. angle & SD & Av. angle & $\mathrm{SD}$ & Av. angle & SD & Av. angle & SD \\
\hline$\overline{P e r 611}$ & 102.09 & 3.34 & 98.7 & 6.33 & 109.96 & 7.57 & 109.74 & 7.46 & 102.90 & 3.63 & 100.81 & 4.89 \\
\hline Per612 & 125.50 & 3.56 & 125.53 & 4.61 & 135.63 & 8.73 & 135.42 & 8.54 & 125.91 & 4.22 & 124.71 & 5.57 \\
\hline Per613 & 133.25 & 2.82 & 131.08 & 4.26 & 135.53 & 6.25 & 135.26 & 6.20 & 133.68 & 2.82 & 130.68 & 4.73 \\
\hline Per614 & 114.03 & 3.16 & 111.24 & 4.47 & 114.04 & 4.16 & 114.08 & 4.11 & 112.30 & 3.01 & 107.62 & 5.40 \\
\hline
\end{tabular}

Analysis of fluctuations (Fig. 2, and visual inspection of trajectories) leads to interesting observation: the "upper" part of PCP is more flexible than the "lower" one (see Fig. 1). All loops are localized in the "upper region". The presence of J7Z lipid affects dynamics of Per611 and Per612, also located in the "upper" region. May be this flexible part (L1: VAL21-GLN36, L2: VAL73-SER81, L3: ILE114-ASN128) should be exploited to have a more elastic hybrid structure (i.e. nano quantum-dot - protein system). If, for some reasons, a more rigid construction is required, the synthetic effort should be directed for the creation of a link in the "lower" part of the PCP system. 


\section{Conclusions}

We have shown that a reasonable computer model of PCP (CHARMM 27 force field) may be constructed. $10 \mathrm{~ns}$ simulation data show good quality of the model. The model will be useful in further studies of PCP protein dynamics, nanomechanics and folding. $10 \mathrm{~ns}$ dynamics of the protein and individual chromophores indicate that the maximum flexibility of PCP is observed in the L2 loop (VAL73-SER81) region and small helices $B, G$ regions (see Fig. 2). Perhaps those areas play an important functional role as hinges during trapping the chromophores in the PCP reconstruction process. The relative orientation dynamics of peridinins and Chl $a$ has been studied. The flexibility of Per chromophores is not identical: two chromophores Per611 and Per614 were found to be more flexible than the other pair. The dynamical data provide us with huge sets of plausible conformations of PCP and such data may be taken into account in the theoretical studies of energy transfer or spectral properties of hybrid systems. It would be interesting to use the present model to study dynamics of Per chromophores in their excited states [23]. It has been found that both Chl $a$ and caroteinoids are necessary to stabilize PCP protein. We plan to perform folding and nanomechanical studies of PCP. The computer model has been prepared for this endeavor.

\section{Acknowledgments}

We thank Dr Anja Damjanovic for providing us bacteriochlorophyll $A$ parameters. The calculations were performed at TASK computer center and were partially supported by a grant no. N202 262038. K.M. acknowledges grant "Krok w przyszłość" from Kujawsko-Pomorskie Voivodeship and Young Scientists Scholarship funded by Institute of Physics NCU. B.K. and S.M. acknowledge support from Foundation for Polish Science Welcome project.

\section{References}

[1] E. Coronado, E. Palomares, J. Mater. Chem. 15, 3593 (2005).

[2] E. Hofmann, P.M. Wrench, F.P. Sharples, R.G. Hiller, W. Welte, K. Diederichs, Science 272, 1788 (1996).

[3] T. Schulte, S. Johanning, E. Hofmann, Eur. J. Cell. Biol. 89, 990 (2010).

[4] T.H. Brotosudarmo, E. Hofmann, R. G. Hiller, S. Wormke, S. Mackowski, A. Zumbusch, C. Brauchle, H. Scheer, FEBS Lett. 580, 5257 (2006).

[5] M. Fuciman, M.M. Enriquez, S. Kaligotla, D.M. Niedzwiedzki, T. Kajikawa, K. Aoki, S. Katsumura, H.A. Frank, J. Phys. Chem. B 115, 4436 (2011).

[6] Z.Q. You, C.P. Hsu, J. Phys. Chem. A 115, 4092 (2011).

[7] T. Schulte, D.M. Niedzwiedzki, R.R. Birge, R.G. Hiller, T. Polivka, E. Hofmann, H.A. Frank, Proc. Nat. Acad. Sci. 106, 20764 (2009).

[8] S. Wormke, S. Mackowski, T. Brotosudarmo, C. Brauchle, A. Garcia, P. Braun, H. Scheer, E. Hofmann, Appl. Phys. Lett. 90, 193901 (2007).
[9] L. Mao, Y. Wang, X. Hu, J. Phys. Chem. B 107, 3963 (2003).

[10] G. Zucchelli, D. Brogioli, A.P. Casazza, F.M. Garlaschi, R.C. Jennings, Biophys. J. 93, 2240 (2007).

[11] I.H.M. van Stokkum, E. Papagiannakis, M. Vengris, J.M. Salverda, T. Polívka, D. Zigmantas, D.S. Larsen, S.S. Lampoura, R.G. Hiller, R. Grondelle, Chem. Phys. 357, 70 (2009).

[12] C. Bonetti, M.T. Alexandre, I.H. van Stokkum, R.G. Hiller, M.L. Groot, R. van Grondelle, J.T. Kennis, Phys. Chem. Chem. Phys. 12, 9256 (2010).

[13] A. Damjanovic, T. Ritz, K. Schulten, Biophys. J. 79, 1695 (2000).

[14] G. Di Paola, L. Guidoni, Eur. Biophys. J. 40, S175 (Suppl 1), (2011).

[15] D. Bovi, A. Mezzetti, R. Vuilleumier, M.P. Gaigeot, B. Chazallon, R. Spezia, L. Guidoni, Phys. Chem. Chem. Phys., DOI: 10.1039/c1cp21985e, (2011).

[16] N. Foloppe, A.D. MacKerell Jr, J. Comput. Chem. 21, 86 (2000)

[17] O.M. Becker, A.D. Mackerell Jr, B. Roux, M. Watanabe, Computational Biochemistry and Biophysics, CRC Press, New York 2001.

[18] A.D. MacKerell, http: //www . pharmacy.umaryland.edu/faculty/ amackere/param/force_field_dev.htm, 2000.

[19] M.J. Frisch, G.W. Trucks, H.B. Schlegel, G.E. Scuseria, M.A. Robb, J.R. Cheeseman, J.A. Montgomery, T. Vreven, K.N. Kudin, J.C. Burant, J.M. Millam, S.S. Iyengar, J. Tomasi, V. Barone, B. Mennucci, M. Cossi, G. Scalmani, N. Rega, G.A. Petersson, H. Nakatsuji, M. Hada, M. Ehara, K. Toyota, R. Fukuda, J. Hasegawa, M. Ishida, T. Nakajima, Y. Honda, O. Kitao, H. Nakai, M. Klene, X. Li, J.E. Knox, H.P. Hratchian, J.B. Cross, V. Bakken, C. Adamo, J. Jaramillo, R. Gomperts, R.E. Stratmann, O. Yazyev, A.J. Austin, R. Cammi, C. Pomelli, J.W. Ochterski, P.Y. Ayala, K. Morokuma, G.A. Voth, P. Salvador, J.J. Dannenberg, V.G. Zakrzewski, S. Dapprich, A.D. Daniels, M.C. Strain, O. Farkas, D.K. Malick, A.D. Rabuck, K. Raghavachari, J.B. Foresman, J.V. Ortiz, Q. Cui, A.G. Baboul, S. Clifford, J. Cioslowski, B.B. Stefanov, G. Liu, A. Liashenko, P. Piskorz, I. Komaromi, R.L. Martin, D.J. Fox, T. Keith, A. Laham, C.Y. Peng, A. Nanayakkara, M. Challacombe, P.M.W. Gill, B. Johnson, W. Chen, M.W. Wong, C. Gonzalez, J.A. Pople, Gaussian 03, Revision C.02, Gaussian, Inc., Wallingford, CT 2004.

[20] V. Zoete, M.A. Cuendet, A. Grosdidier, O. Michielin, J. Comput. Chem. 32, 2359 (2011).

[21] J.C. Phillips, R. Braun, W. Wang, J. Gumbart, E. Tajkhorshid, E. Villa, C. Chipot, R.D. Skeel, L. Kale, K. Schulten, J. Comput. Chem. 26, 1781 (2005).

[22] F.J. Kleima, E. Hofmann, B. Gobets, I.H. van Stokkum, R. van Grondelle, K. Diederichs, H. van Amerongen, Biophys. J. 78, 344 (2000).

[23] R. Simonetto, M. Crimi, D. Sandona, R. Croce, G. Cinque, J. Breton, R. Bassi, Biochemistry 38, 12974 (1999). 